PREPARED FOR THE U.S. DEPARTMENT OF ENERGY, UNDER CONTRACT DE-AC02-76CH03073

PPPL-3636

PPPL-3636

UC-70

Fluctuation Measurements in Tokamaks with Microwave Imaging Reflectometry

by

E. Mazzucato, T. Munsat, H. Park, B.H. Deng, C.W. Domier N.C. Luhmann, Jr., A.J.H. Donné, and M.J. van de Pol

December 2001

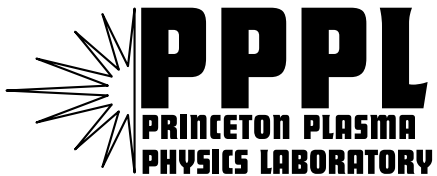

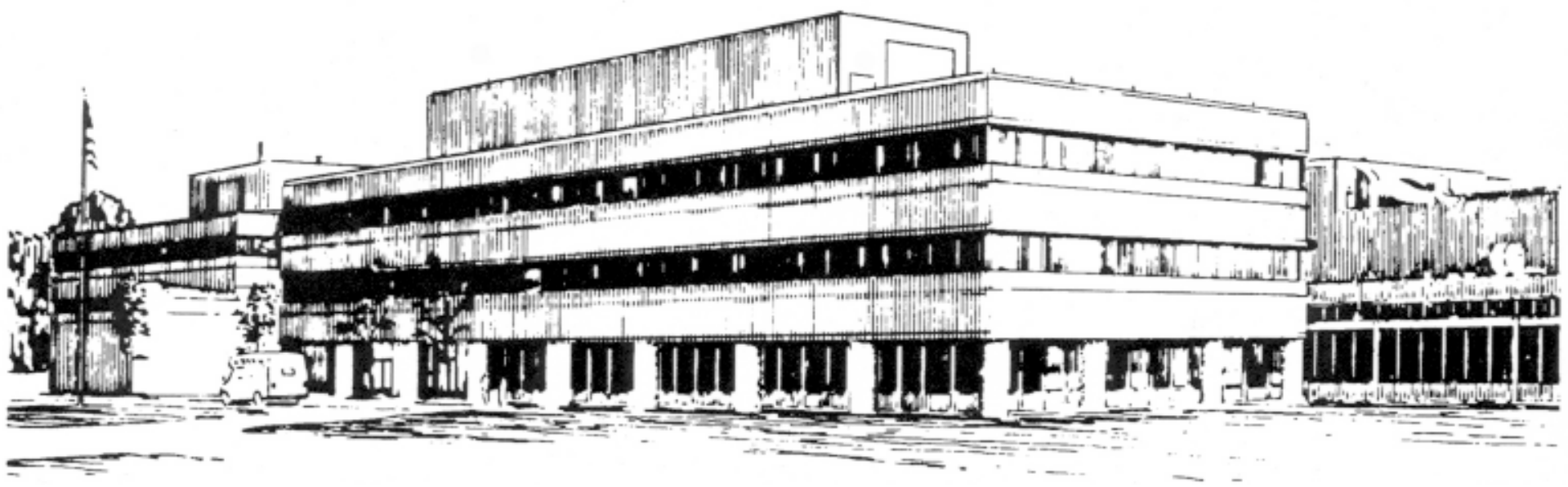

PRINCETON PLASMA PHYSICS LABORATORY PRINCETON UNIVERSITY, PRINCETON, NEW JERSEY 


\section{PPPL Reports Disclaimer}

This report was prepared as an account of work sponsored by an agency of the United States Government. Neither the United States Government nor any agency thereof, nor any of their employees, makes any warranty, express or implied, or assumes any legal liability or responsibility for the accuracy, completeness, or usefulness of any information, apparatus, product, or process disclosed, or represents that its use would not infringe privately owned rights. Reference herein to any specific commercial product, process, or service by trade name, trademark, manufacturer, or otherwise, does not necessarily constitute or imply its endorsement, recommendation, or favoring by the United States Government or any agency thereof. The views and opinions of authors expressed herein do not necessarily state or reflect those of the United States Government or any agency thereof.

\section{Availability}

This report is posted on the U.S. Department of Energy's Princeton Plasma Physics Laboratory Publications and Reports web site in Fiscal Year 2002. The home page for PPPL Reports and Publications is: http://www.pppl.gov/pub_report/

DOE and DOE Contractors can obtain copies of this report from:

U.S. Department of Energy

Office of Scientific and Technical Information

DOE Technical Information Services (DTIS)

P.O. Box 62

Oak Ridge, TN 37831

Telephone: (865) 576-8401

Fax: (865) 576-5728

Email: reports@adonis.osti.gov

This report is available to the general public from:

National Technical Information Service

U.S. Department of Commerce

5285 Port Royal Road

Springfield, VA 22161

Telephone: 1-800-553-6847 or

(703) 605-6000

Fax: (703) 321-8547

Internet: http://www.ntis.gov/ordering.htm 


\title{
Fluctuation measurements in tokamaks with microwave imaging reflectometry
}

E. Mazzucato ${ }^{\text {a) }}$, T. Munsat, and H. Park

Princeton Plasma Physics Laboratory, Princeton, New Jersey 08543, USA

B.H. Deng, C.W. Domier, and N.C. Luhmann, Jr.

Department of Applied Sciences, University of California at Davis, Davis, California 95616, USA

A.J.H. Donné, and M.J van de Pol

FOM-Instituut voor Plasmafysica Rijnhuizen, Associatie EURATOM-FOM,

P.O. Box 1207, 3430 BE Nieuwegein, The Netherlands

\begin{abstract}
To study the mechanism of anomalous transport in tokamaks requires the use of sophisticated diagnostic tools for the measurement of short-scale turbulent fluctuations. In this article, we describe an attempt at developing a technique capable of providing a comprehensive description of plasma fluctuations with $k_{\perp} \rho_{i}<1$, such as those driven by the Ion Temperature Gradient mode in tokamaks. The proposed method is based on microwave reflectometry, and stems from a series of numerical calculations showing that the spatial structure of fluctuations near the cutoff could be obtained from the phase of reflected waves when these are collected with a wide aperture optical system forming an image of the cutoff onto an array of phase sensitive detectors. Preliminary measurements with a prototype apparatus on the Torus Experiment for Technology Oriented Research 94 (TEXTOR-94) [U. Samm, Proceedings of the 16th IEEE Symposium on Fusion Engineering, 1995 (IEEE, Piscataway, NJ, 1995), p. 470] confirm the validity of these conclusions. Technical issues in the application of the proposed technique to tokamaks are discussed in this article, and the conceptual design of an imaging reflectometer for the visualization of turbulent fluctuations in the National Spherical Torus Experiment (NSTX) [M. Ono, et al., Nucl. Fusion 40, 557 (2000)] is described.
\end{abstract}

Key words: Tokamak, anomalous transport, plasma turbulence, turbulent fluctuations, microwave imaging reflectometry.

\footnotetext{
a) Electronic mail: mazzucato@pppl.gov
} 


\section{INTRODUCTION}

Understanding the mechanism of anomalous transport in tokamaks is one of the great challenges of fusion research. Indeed, since most explanations of this phenomenon are based on some type of turbulence,,$^{1,2}$ understanding anomalous transport is tantamount to understanding plasma turbulence.

In tokamak research, a longstanding conjecture is that the observed anomalous plasma transport is caused by some type of drift wave instability. Accordingly, for turbulent fluctuations that are isotropic perpendicularly to the magnetic field, the energy replacement time $\left(\tau_{E}\right)$ must exhibit a Bohm scaling $\left[\tau_{E} \propto \Omega^{-1}\left(a / \rho_{i}\right)^{2}\right]$ when $k_{\perp} \propto a^{-1}$, and a GyroBohm scaling $\left[\tau_{E} \propto \Omega^{-1}\left(a / \rho_{i}\right)^{3}\right]$ when $k_{\perp} \propto \rho_{i}^{-1}$ (here and in the following, $a$ is the plasma minor radius, $k_{\perp}$ is the fluctuation wave number perpendicularl to the magnetic field, $\Omega$ is the ion cyclotron frequency and $\rho_{i}$ is the ion Larmor radius). For anisotropic turbulence, predictions become more complex. For instance, $\tau_{E}$ can follow a Gyro-Bohm scaling for $k_{\theta} \propto a^{-1}$ and $k_{r} \propto\left(a \rho_{i}\right)^{-1 / 2}$ (where $k_{\theta}$ and $k_{r}$ are the poloidal and radial components of $k_{\perp}$, respectively).

Empirical scalings of $\tau_{E}$ tend to support a Gyro-Bohm scaling, even though they all contain other dimensionless parameters besides the normalized Larmor radius $\rho^{*}\left(=\rho_{i} / a\right)$. A case in point is that used to predict the performance of the International Thermonuclear Experimental Reactor, ${ }^{3}$ which in standard notation is $\tau_{E} \Omega \propto \rho^{*-2.70} \beta^{-0.90} v^{*-0.01} M^{0.96} q_{95}^{-3.0} A^{-0.73} \kappa^{2.3}$. Unfortunately, such empirical scalings find meager support in the existing data base of turbulence measurements.

The main difficulty in drawing any firm conclusion from fluctuation measurements is their scarcity and limitations. For example, wave scattering measurements, ${ }^{4-8}$ which were so prominent in early fluctuation studies, have a poor spatial resolution - in most cases larger than the plasma minor radius. The method of Beam Emission Spectroscopy 9 requires a perturbing neutral beam and has serious difficulties in detecting plasma fluctuations in the central core of large tokamaks. The interpretation of microwave reflectometry is extremely difficult, and in the best of cases it cannot be done unambiguously. ${ }^{10-12}$ The inevitable conclusion is that, for advancing our understanding of turbulence, we must improve the capability of our diagnostic tools. 
In this article, we describe a novel technique for the global visualization of short-scale fluctuations with $k_{\perp} \rho_{i}<1$ in the main core of tokamak plasmas. The outline is as follows. In Sec. II, the intrinsic difficulties and limitations in the use of standard reflectometry in tokamaks are discussed and explained using the results of a series of numerical simulations. The method of Microwave Imaging Reflectometry is described in Sec. III together with a discussion of technical issues for its use in tokamaks. Results from the first use of imaging reflectometry are presented in Sec. IV, and the conclusions are given in Sec. V.

\section{NUMERICAL RESULTS}

The method of Microwave Imaging Reflectometry ${ }^{13}$ described in this article is a technique for the visualization of density fluctuations with $k_{\perp} \rho_{i}<1$, such as those driven by the Ion Temperature Gradient (ITG) mode. It is based on microwave reflectometry ${ }^{12}-\mathrm{a}$ radar technique for the detection of plasma density fluctuations using the reflection of microwaves from a plasma cutoff. Because of the high sensitivity to plasma fluctuations, microwave reflectometry has found extensive use for the detection of turbulence in tokamaks. Unfortunately, very often the high sensitivity makes it very difficult to extract any quantitative information from the measured signals. The root of the problem is illustrated in Figs. 1(a) and 1(b), which show schematically the main difference between reflectometry in a 1D and 2D geometry, the latter being obviously the case of interest for tokamaks.

The interpretation of reflectometry in a 1D geometry [Fig. 1(a)] is relatively simple. In this case, a plane stratified plasma permittivity $\varepsilon=\varepsilon_{0}(r)+\tilde{\varepsilon}(r)$ [with fluctuation component $\tilde{\varepsilon}(r)<<1]$ is probed by a wave propagating in the $r$-direction. Under these conditions, it is easy to show that, when the radial wave number of fluctuations satisfies the condition $k_{r}<k_{0} /\left(k_{0} L_{\varepsilon}\right)^{1 / 3}$ [where $L_{\varepsilon}=\left(d \varepsilon_{0} / d r\right)_{r=r_{c}}^{-1}$ is the scale length of the plasma permittivity at the cutoff $r=r_{c}$ and $k_{0}$ is the free-space wave number of the probing beam], the fluctuating phase of the reflected signal is given by the approximation of geometric optics ${ }^{12}$

$$
\tilde{\phi}=k_{0} \int_{0}^{r_{c}} \frac{\tilde{\varepsilon}(r)}{\sqrt{\varepsilon_{0}(r)}} d r .
$$

Taking $\left|k_{r}\right|>1 / L_{\varepsilon}$ (since we are interested in short-scale fluctuations) and $\varepsilon_{0}(r) \approx\left(r_{c}-r\right) / L_{\varepsilon}$ (since most of the contribution to $\tilde{\phi}$ comes from a narrow region near 
the cutoff), for $\left(2\left|k_{r}\right| L_{\varepsilon} / \pi\right)^{1 / 2}>>1$ we obtain 10,12

$$
\Gamma_{\phi}\left(k_{r}\right)=\pi M \frac{k_{0}^{2} L_{n}}{\left|k_{r}\right|} \Gamma_{n}\left(k_{r}\right),
$$

where $L_{n}=n /(d n / d r)_{r=r_{c}}$ is the scale length of the electron density $n, M \equiv(n \partial \varepsilon / \partial n)_{r=r_{c}}$ $(\approx 1$ for the ordinary mode and $\approx 2$ for the extraordinary mode $), \Gamma_{\phi}\left(k_{r}\right)$ is the power spectrum of $\tilde{\phi}$ (considered as a function of $r_{c}$ ) and $\Gamma_{n}\left(k_{r}\right)$ is the power spectrum of the relative plasma density fluctuation $\tilde{n} / n$. In summary, the power spectrum of $1 \mathrm{D}$ fluctuations $\left(\Gamma_{n}\right)$ can be obtained from the power spectrum of the signal phase $\left(\Gamma_{\phi}\right)$. The latter can be obtained with radial correlation measurements using several probing waves with closely spaced cutoff layers.

The interpretation of reflectometry becomes considerably more complicated in the case of multidimensional fluctuations where, as in the case of tokamaks, the plasma permittivity varies perpendicularly to the direction of propagation of the probing wave [Fig. 1(b)]. In this case, the measured backward field cannot be described as a specular reflection of the probing wave, and, more importantly, its properties may differ drastically from those of plasma fluctuations. This can be easily seen by taking a $2 \mathrm{D}$ wave permittivity $\varepsilon=\varepsilon_{0}(r)+\tilde{\varepsilon}(r, x)$ and assuming that near the cutoff the reflected wave can be cast in the form $\exp (i \tilde{\phi}(x))$, with $\tilde{\phi}(x)$ given by Eq. (1). Since the phase of the probing wave is the cumulative result of many random contributions, we may assume that $\tilde{\phi}$ is a normal random variable with mean $\left\langle\tilde{\phi}>=0\right.$, variance $\sigma_{\phi}^{2} \equiv<\tilde{\phi}^{2}>$ and autocorrelation $\gamma_{\phi}(\xi) \equiv<\tilde{\phi}_{1}(x) \tilde{\phi}_{2}(x+\xi)>/ \sigma_{\phi}^{2}$. From this, we find that the first moment of the wave electric field (i.e., the amplitude the coherent specular reflection) is $\langle E\rangle=\exp \left(-\sigma_{\phi}^{2} / 2\right)$, and thus it is a decreasing function of $\sigma_{\phi}$. For the second moment, we obtain $<E_{1} E_{2}^{*}>=\exp \left[-\sigma_{\phi}^{2}\left(1-\gamma_{\phi}\right)\right]$, which shows that the signal correlation length is also a decreasing function of $\sigma_{\phi}$. In particular, for $\sigma_{\phi}>>1$, taking $\gamma_{\phi}(\xi)=\exp \left[-(\xi / \Delta)^{2}\right]$ and expanding to the second order in $\xi$, we obtain $\left\langle E_{1} E_{2}^{*}>\approx \exp \left[-\left(\sigma_{\phi} \xi / \Delta\right)^{2}\right]\right.$. Therefore, in the presence of large 2D density fluctuations, the spectrum of reflected waves becomes broader than the spectrum of $\tilde{\phi}$ by a factor of $\sigma_{\phi}$. Consequently, if $\Delta k_{x}$ is the width of fluctuations in the $x$-direction and $\sigma_{\phi} \Delta k_{x}<<k_{0}$, the reflected waves are spread over the range of wave numbers $\delta k_{r} \approx \sigma_{\phi}^{2} \Delta k_{x}^{2} / 2 k_{0}$ [Fig. 1(b)], so that an observer at a distance from the cutoff that is larger than $1 / \delta k_{r}$ (as in large tokamak experiments) will sample a complicated interference pattern, with large amplitude variations and random phases. This suggests that for a signal with unit average power, its amplitude $\rho$ must follow the 
distribution derived by Rice $^{14}$ for the case of a signal containing a coherent sinusoidal component and a Gaussian noise, which is given by

$$
P(\rho)=\frac{\rho}{\sigma^{2}} \mathrm{e}^{-\left(\rho^{2}+\rho_{0}^{2}\right) / 2 \sigma^{2}} I_{0}\left(\frac{\rho \rho_{0}}{\sigma^{2}}\right),
$$

where $I_{0}$ is the modified Bessel function of order zero, $\rho_{0}$ is the amplitude of the coherent component, and $\sigma^{2}\left(=\left(1-\rho_{0}^{2}\right) / 2\right)$ is the variance of both the real and the imaginary parts of the Gaussian noise. For large fluctuations with $\sigma_{\phi}^{2}>>1, \rho_{0} \approx 0$ [since $\rho_{0}^{2} \approx \exp \left(-\sigma_{\phi}^{2}\right)$ ] and Eq. (3) becomes the Rayleigh distribution $2 \rho \exp \left[-\rho^{2}\right]$. This result, which we have derived from simple and somewhat arbitrary assumptions, appears to describe very well the experimental observed behavior of reflectometry signals from large tokamaks, as demonstrated in Fig. 2 where the in-phase $(I)$ and quadrature $(Q)$ components of a reflectometer signal from a Tokamak Fusion Test Reactor (TFTR) plasma are shown together with the density distribution of the amplitude $\rho=\sqrt{I^{2}+Q^{2}}$. Both the $I / Q$ plot and the amplitude density distribution in Fig. 2 are consistent with the above arguments if we assume $\sigma_{\phi}^{2} \gg 1$.

If it is true that the problem of standard reflectometry arises from the interference of reflected waves at the point of measurement, Fig. 1(c) suggests that the remedy is to collect the reflected waves with a wide aperture optical system and to form an image of the cutoff onto the detector plane. This conjecture was tested with a series of numerical simulations ${ }^{13,15}$ using a plane-stratified plasma equilibrium with density $n(r)$ and a field of 2D density fluctuations $\tilde{n}(r, x)$ ( $r$ and $x$ representing the radial and poloidal coordinates of a tokamak configuration). In the following, we summarize the major results obtained using a TFTR-like density profile and a probing wave with a frequency of $75 \mathrm{GHz}$ and the ordinary mode of propagation.

The first result is that at the plasma boundary, similarly to the experimental case of Fig. 2 , the reflected waves exhibit large and random phase and amplitude modulations [Figs. 3 (a) and 4(a)]. The case shown in these figures is that of 2D turbulent fluctuations with a nearly Gaussian spectrum having a poloidal width $\Delta k_{\theta}=0.5 \mathrm{~cm}^{-1}$, a radial width $\Delta k_{r}=1.0$ $\mathrm{cm}^{-1}$ and a total density fluctuation $\tilde{n} / n=1 \%$. For comparison, the dotted line in Fig. 4 shows the phase of 1D geometric optics [Eq. (1)], which is our only tool for obtaining a measure of the plasma density fluctuation from reflectometry signals. Figure 4(a) demonstrates that this is not possible when signals are detected at a large distance from the plasma cutoff, as in most reflectometry experiments. However, as suggested by Fig. 1(c), 
one could sample the field of reflected waves at other locations by creating an image onto the plane of measurement with an optical system. Numerically, this can be easily accomplished by projecting back in space the solution of the wave equation. Our numerical simulations indicate [Figs. 3(b) and 4(b)] that fluctuations in the amplitude of the backward field are minimum on a plane (virtual cutoff) behind the cutoff, where the fluctuating part of the phase is identical to the phase of Eq. (1). The displacement of the virtual cutoff from the real cutoff is explained by the fact that the reflected waves propagate in a medium with refractive index less than unity. Finally, as shown in Fig. 4(c), we find that the phase of Eq. (1) is almost identical to the normalized density fluctuation in front of the real cutoff, i.e., where the spatial structure of the probing wave ( Airy function) has its maximum value. For the case of Fig. 4, this occurs at approximately $1 \mathrm{~cm}$ in from the cutoff.

In conclusion, these results could be summarized by saying that the field of reflected waves arises near the cutoff from a phase modulation of the probing wave, with a magnitude given by 1D geometric optics. Since the reflected waves propagate in a nonuniform medium with permittivity less than unity, the backward field appears to arrive from a distant point behind the cutoff, where it can be approximated by a plane wave $E=\exp [i \tilde{\phi}(x)]$, with $\tilde{\phi}$ given by Eq. (1). The problem of 2D fluctuations has been reduced to a 1D problem, and data on plasma fluctuations can be obtained with the use of imaging optics.

Obviously, this simple description of reflectometry must fail for large fluctuations. In fact, we can easily derive two conditions for its validity. The first condition is obtained from the criterion for the validity of 1D geometric optics, ${ }^{12}$ given by $\Delta k_{r}<k_{0} /\left(k_{0} L_{\mathcal{\varepsilon}}\right)^{1 / 3}$. The second condition can be derived from the fact that the field of reflected waves arises from the nonlinear coupling of the various mode of propagation. Since for each spectral component of the backward field this occurs mainly near the corresponding reflecting point, our model of reflectometry must fail when the turning points of reflected waves are distributed over a distance $\Delta r_{c}$ that is comparable to the radial scale length of fluctuations $\left(\Delta k_{r}^{-1}\right)$, i.e., when $\Delta k_{r} \Delta r_{c}>1$. Since for large fluctuations (i.e., $\sigma_{\phi}^{2}>>1$ ) $\Delta r_{c} / L_{\varepsilon} \approx \sigma_{\phi}^{2} \Delta k_{\theta}^{2} / k_{0}^{2}$, this condition becomes $\sigma_{\phi}^{2}<k_{0}^{2} / L_{\varepsilon} \Delta k_{r} \Delta k_{\theta}^{2}$, which can be cast in the form

$$
\frac{\tilde{n}}{n}<\frac{1}{\pi^{3 / 4} L_{n} \Delta k_{\theta}}
$$


by using Eq. (2) [valid for $\left(2\left|k_{r}\right| L_{\varepsilon} / \pi\right)^{1 / 2} \gg>1$ ]. This condition is satisfied by the fluctuation parameters in Fig. 4 (where $L_{n}=50 \mathrm{~cm}$ ). On the other hand, for slightly different fluctuations parameters $\left(\Delta k_{\theta}=1.0 \mathrm{~cm}^{-1}, \Delta k_{r}=1.0 \mathrm{~cm}^{-1}, \tilde{n} / n=1.5 \%\right)$ not satisfying Eq. (4), we obtain the results of Fig. 5 showing a backward field that is affected everywhere by large amplitude and phase fluctuations.

\section{MICROWAVE IMAGING REFLECTOMETRY}

In this section, we discuss some technical issues in the application of microwave imaging reflectometry to tokamaks. Figure 6 illustrates schematically the basic imaging scheme, where the probing and reflected beams enter and exit the vacuum chamber through the same window, and share a common set of primary optics. The reflected beam is separated from the probing beam with a beam splitter, and is focused onto an array of detectors.

Though the primary optics are shared between the probing and the reflected beam, the optical elements do not perform the same function during illumination and detection. For the probing wave, their role is to tailor the wave front to the shape of the cutoff surface, thus making the wave rays impinge perpendicularly upon the cutoff surface, thereby minimizing the deleterious effect of plasma refraction on the spectrum of probing wave numbers. ${ }^{13}$ To perform this function, the optical system must have two different focal points, one located at the center of the torus in the equatorial plane, the second at the center of curvature of the cutoff surface in the poloidal plane. It should also be noted that without this curvature matching, any small deviation in the radial position of the cutoff would cause large deviation of the reflected beam, and degradation of the spectrum of collected radiation. By using the curvature-matching technique, cutoff surfaces over a wide range of densities can be illuminated with minimal changes to the illumination optics.

For the reflected wave, the function of the optical system is that of creating an image of the virtual cutoff onto an array of detectors. This is illustrated in Fig. 6(b), which shows the reflected beam from three points of the virtual cutoff (located behind the actual cutoff as described in Sec. II). To overcome the limitation on the amount of power available for the probing beam, it may be convenient to use additional optics (not shown in Fig. 6) for demagnifying the image as much as possible consistently with the detector element size. 
Furthermore, additional optics may also be needed to compensate for the two different focal points of the primary optics.

In a realistic diagnostic configuration, the main optical limitation will be imposed by the size of the vacuum window. Assuming that the rest of the optical system does not vignette the reflected beam, and a Gaussian radiation profile for the detectors, the best instrument resolution will be given by the Gaussian "spot-size" at the cutoff, given by $2 \delta=2 \lambda d / \pi r$ (where $\delta$ is the Gaussian beam waist at the object plane, $\lambda$ is the wavelength of the probing beam, $d$ is the distance from the aperture to the beam waist, and $r$ is the window radius). Clearly, it is possible for the instrument resolution to be different in the toroidal and poloidal directions. Furthermore, the resolution of off-axis channels could be degraded by further vignetting of the reflected signal.

The amount of coverage in the plasma, which also determines the lower limit of the $k_{\theta}$ resolution, will also be constrained by the window size. This is obvious from Fig. 6(a), which shows how the size and location of the window relative to the cutoff surface determine the illuminated plasma region. A further limitation is that, for avoiding diffraction from the window edge, the beam profile must fall off near the window edges.

The viability of imaging reflectometry is dependent on the availability of sensitive detectors. In this regard, there has been steady progress in the area of millimeter-wave detectors, particularly in the design of arrays that could be inexpensively manufactured on printed circuit boards, and therefore could be scaled up to large multichannel arrays. ${ }^{16}$

Figure 7 illustrates the conceptual design of a microwave imaging reflectometer for the National Spherical Torus Experiment (NSTX), a low aspect ratio tokamak with the mission of investigating the physics of high beta plasmas. ${ }^{17}$ Some of its parameters are major radius $0.85 \mathrm{~m}$, minor radius $0.67 \mathrm{~m}$, toroidal magnetic field 0.3-0.45 T, plasma current 0.71.4 MA, and central temperatures $0.5-2.0 \mathrm{keV}$.

Compared to the scheme of Fig. 6, the major innovation in the design of Fig. 7 is the use of reflective optics for avoiding the spurious effects of internal reflections in refractive optics. Figure 7(a) shows the ray trajectories (dashed lines) of a $65 \mathrm{GHz}$ probing beam (Xmode) together with the trajectories (solid lines) of the reflected wave in the absence of plasma fluctuations. The coincidence of these two sets of trajectories demonstrates a nearly perfect matching of the probing wave front to the cutoff curvature. Figure 7(b) shows the ray trajectories of six backward waves that are born on the equatorial plane (near the cutoff) with a wave number $\leq 2.0 \mathrm{~cm}^{-1}$ (to be compared with $0.5 \mathrm{~cm}^{-1}$ for the poloidal 
wave number of expected fluctuations in NSTX plasmas). These waves are focused on a single point of the detector plane - the image of the virtual cutoff. The position of the latter is indicated in Fig. 7(b) by the intersection of the dashed lines. The illuminated plasma area is $\sim 25 \mathrm{~cm}$ tall, resulting in a lower limit of $0.25 \mathrm{~cm}^{-1}$ for the wave number resolution. Finally, the spatial resolution in the poloidal direction is $\sim 0.5 \mathrm{~cm}$.

\section{PRELIMINARY RESULTS}

Recently, the first experimental application of imaging reflectometry to tokamaks has been made on TEXTOR-94 ${ }^{18}$ (Torus Experiment for Technology Oriented Research 94) a circular cross section tokamak with $a=0.50 \mathrm{~m}, R=1.75 \mathrm{~m}$, and $B \leq 2.9 \mathrm{~T}$. The reflectometer was built using the optical components of an electron cyclotron emission (ECE) imaging apparatus, which was used previously for the study of temperature fluctuations in TEXTOR-94. ${ }^{19,20}$ Indeed, this underscores one of the main advantages of imaging reflectometry, i.e., the possibility of sharing the optics with an ECE imaging system, and thus making possible a tool for the simultaneous visualization of both density and temperature fluctuations. The development of such a system is the goal of an ongoing collaboration between the University of California at Davis, the Princeton Plasma Physics Laboratory and the FOM Institute for Plasma Physics.

The prototype imaging reflectometer, whose scheme was basically that shown in Fig. 6, shared the vacuum window with the Thomson scattering apparatus. In this experiment, both the probing frequency ( $84 \mathrm{GHz}, \mathrm{X}$-mode) and the focal plane of the optics were held fixed, and the electron density was ramped during the shot to bring the cutoff surface through and beyond the focal plane of the optics. Figure 8 shows some $I / Q$ plots from a single channel recorded over several $3 \mathrm{~ms}$ time windows as the central plasma density changed from $2.8 \times 10^{19} \mathrm{~m}^{-3}$ at $\mathrm{t}=1.2 \mathrm{~s}$ [Fig. 8(a)], to $4.4 \times 10^{19} \mathrm{~m}^{-3}$ at t=2.4 s [Fig. 8(d)].

During the density ramp, the cutoff position moved from 1.93 to $2.06 \mathrm{~m}$ [with an intermediate position of $1.99 \mathrm{~m}$ at the time of both plots (b) and (c)].

The striking difference between plots $(a, d)$ and $(b, c)$ is in the level of amplitude fluctuations, which is much larger in the former case. These data were collected in ohmic plasmas where, apart from a small density rise, all plasma parameters were stationary. Thus, consistent with the numerical results of Fig. 3, we attribute the much smaller level of amplitude fluctuations of plots $(b, c)$ to the in-focus condition of the virtual cutoff. This is 
confirmed by the agreement between the numerical estimate of its position in plots (b,c) with the radial position of the focal plane, and by the fact that the distance between the cutoffs of (a) and (b) or between those of (c) and (d) is larger than the optical depth of focus.

The histograms of the signal amplitude are displayed in Fig. 9 together with the best fit to the Rice probability distribution [Eq. (3)]. This shows that the amplitude of the coherent reflection goes from zero during out-of-focus conditions, to close to unity when the virtual cutoff is in-focus. The fact that the Rice distribution appears to describe the distribution of amplitudes even during the in-focus conditions can be explained by a residual of amplitude fluctuations, as shown by the numerical simulations [Fig. 3(b)], and by a nonperfect focusing of the cutoff because of optical aberrations.

Another remarkable difference between the in-focus and the out-of-focus conditions is the time evolution of the phase of measured signals. This is illustrated in Fig. 10, which shows that during out-of-focus conditions the average rate of change is more than two orders of magnitude larger than during in-focus conditions. This phenomenon, which cannot be explained with the rise in density, is known in standard reflectometry as the runaway-phase phenomenon. Similarly, the power spectra are also very different in the two cases, as illustrated by Fig. 11 where the phase power spectrum is dominated by large coherent MHD fluctuations when the cutoff is the in-focus, while it becomes a featureless $1 / f^{2}$ spectrum when the cutoff goes out-of-focus. What is remarkable is that in the latter case, the spectrum does not give the slightest hint of the presence of large MHD fluctuations. Again, this is a known phenomenon in standard reflectometry, ${ }^{12}$ whose cause has always been a matter of conjecture. This effect appears to be fully explained by our measurements.

These measurements confirm the validity of our numerical results (Sec. II) that are at the basis of microwave imaging reflectometry, and clearly demonstrate the advantages of this technique over standard reflectometry - regardless of whether the measurement is a single or a multi-point measurement. The fact that the introduction of focusing optics allows multiple channels measurements must be considered just a fringe benefit of the proposed method. 


\section{CONCLUSION}

In summary, our numerical results support the conjecture that the chaotic behavior of signals in standard reflectometry is due to the interference of reflected waves at the point of measurement. This is caused by the scattering of reflected waves over a large angle by the 2D structure of tokamak fluctuations. The numerical results suggest that a mapping of fluctuations near the cutoff could be obtained from the phase of measured signals if the reflected waves are collected with a wide aperture antenna, and an image of the cutoff is made onto an array of microwave detectors (taking plasma refraction into account). These conclusions appear to be fully confirmed by measurements on TEXTOR-94 using a prototype imaging reflectometer.

These results form the basis of an apparatus - currently under construction - that will be used on TEXTOR-94 for the study of turbulent fluctuations in plasmas with a dynamic ergodic divertor. The reflectometer will operate in the frequency range $84-90 \mathrm{GHz}$ using the optical scheme of Fig. 7, which will be shared with an electron cyclotron imaging system ${ }^{20}$ operating in the frequency range $110-140 \mathrm{GHz}$. A dichroic plate will be used to separate the reflectometer signal from the plasma cyclotron emission.

In conclusion, the method described in this article must be considered an attempt at developing techniques for the global visualization of turbulent and coherent structures with microwave reflectometry. Undoubtedly, its practical implementation presents serious difficulties, such as the need for large machine ports and 2D arrays of microwave detectors. Nevertheless, this technique has the potential for providing new and important information on the spatial structure of turbulent fluctuations in tokamaks and spherical tori. 


\section{ACKNOWLEDGEMENT}

This work was supported by U.S. DOE Contract No. DE-AC02-76-CHO-3073, and by NWO and EURATOM under the EURATOM-FOM association agreement.

\section{REFERENCES}

${ }^{1}$ W. Horton, Rev. Mod. Phys. 71, 735 (1999).

2J. W. Connor and H. R. Wilson, Plasma Phys. Control. Fusion 36, 719 (1994).

${ }^{3}$ ITER Physics Expert Groups on Confinement and Transport, Nucl. Fusion 39, 2175 (1999).

${ }^{4}$ E. Mazzucato, Phys. Rev. Lett.. 36, 792 (1976).

${ }^{5}$ C. M. Surko and R. E. Slusher, Phys. Rev. Lett.. 37, 1747 (1976).

${ }^{6}$ D. L. Brower, W. A. Peebles, and N. C. Luhmann, Jr., Nucl. Fusion 27, 2055 (1987).

${ }^{7}$ R. Philipona, E. J. Doyle, N. C. Luhmann, Jr., W. A. Peebles, C. Rettig, K. H. Burrell, R. J. Groebner, H. Matsumoto, and the DIII-D Group, Rev. Sci. Instrum. 61, 3007 (1990).

${ }^{8}$ P. Devynck, X. Garbet, C. Laviron, J. Payan, S. K. Saha, F. Gervais, P. Hennequin, A. Quéméneur, and A. Truc, Plasma Phys. Control. Fusion 35, 63 (1993).

${ }^{9}$ R. Fonck, G. Cosby, R. D. Durst, S. F. Paul, N. Bretz, S. Scott, E. Synakowski, and G. Taylor, Phys. Rev. Lett.. 70, 3736 (1993).

${ }^{10}$ E. Mazzucato and R. Nazikian, Phys. Rev. Lett. 71, 1840 (1993).

${ }^{11}$ R. Nazikian and E. Mazzucato, Rev. Sci. Instrum. 66, 392 (1995).

${ }^{12}$ E. Mazzucato, Rev. Sci. Instrum. 69, 2201 (1998).

${ }^{13}$ E. Mazzucato, Nucl. Fusion 41, 203 (2001).

${ }^{14}$ S. O. Rice, Bell System Tech. J., 23, 282 (1944); 24, 96 (1945) (reprinted in N. Wax, Selected Papers on Noise and Stochastic Processes (Dover, New York, 1954)).

${ }^{15}$ E. Mazzucato, Rev. Sci. Instrum. 69, 1691 (1998).

${ }^{16}$ P.L. Hsu, B.H. Deng, J. Wang, C.W. Domier, and N.C. Luhmann, Jr, Rev. Sci. Instrum 72, 364 (2001).

${ }^{17}$ M. Ono, S. M. Kaye, M. Peng et al., Nucl. Fusion 40, 557 (2000). 
${ }^{18}$ U. Samm, Proceedings of the 16th IEEE Symposium on Fusion Engineering, 1995 (IEEE, Piscataway, NJ, 1995), p. 470

${ }^{19}$ A. J. H. Donné, R. Jaspers, C. J. Barth et al. Rev. Sci. Instrum. 72, 1046 (2001).

${ }^{20}$ B.H. Deng, C. W. Domier, N. C. Luhmann, Jr., et al., Rev. Sci. Instrum. 72, 301 (2000) 


\section{FIGURES CAPTIONS}

Figure 1. Schematic representation of standard reflectomery in 1D $(a)$ and 2D (b) geometry, and that of imaging reflectometry $(c)$.

Figure 2. In-phase $(I)$ and quadrature $(Q)$ components of a TFTR reflectometer signal (left) and probability density distribution of the amplitude $\rho=\left(I^{2}+Q^{2}\right)^{1 / 2}$ (right). Dashed line is the Rayleigh distribution.

Figure 3. Plot of the complex amplitude of reflected waves at the plasma edge (a) and at the virtual cutoff $(b)$ for $\Delta k_{\theta}=0.5 \mathrm{~cm}^{-1}, \Delta k_{r}=1.0 \mathrm{~cm}^{-1}$, and $\tilde{n} / n=1.0 \%)$.

Figure 4. Phase fluctuation of reflected signals at the plasma boundary $(a)$ and the virtual cutoff $(b)$, and normalized density fluctuation near the cutoff $(c)$; dashed lines are the phase of 1D geometric optics $\left(\Delta k_{\theta}=0.5 \mathrm{~cm}^{-1}, \Delta k_{r}=1.0\right.$ $\mathrm{cm}^{-1}$, and $\tilde{n} / n=1.0 \%$.).

Figure 5. Plot of the complex amplitude of reflected waves at the plasma edge (a) and at the virtual cutoff $(b)$ for $L_{n}=50 \mathrm{~cm}, \Delta k_{\theta}=1.0 \mathrm{~cm}^{-1}, \Delta k_{r}=1.0 \mathrm{~cm}^{-1}$, and $\tilde{n} / n=1.5 \%$.

Figure 6. Schematic diagram of an imaging reflectometer showing probing (a) and reflected beams (b).

Figure 7. Conceptual design of an imaging reflectometer for NSTX; $\mathrm{S}$ is the microwave source, M1 and M2 are cylindrical mirrors. Ray trajectories are from a ray tracing code including plasma refraction.

Figure 8. Quadrature signals plots (normalized to unit average power) over a $3 \mathrm{msec}$ time window as the cutoff moves through the optical focal plane because of a density rise. Cutoff positions are $1.93 \mathrm{~m}(a), 1.99 \mathrm{~m}(b, c)$, and $2.06 \mathrm{~m}(d)$.

Figure 9. Amplitude histograms of the signals of Fig. 9 and best fit to a Rice distribution (smooth lines). The Rice parameters $\left[\rho_{0}, \sigma\right]$ are $[0.00,0.71](a),[0.95,0.22]$ $(b),[0.98,0.14](c),[0.00,0.71](d)$.

Figure 10. Time evolution of the signal phase during out-of-focus (a) and in-focus (b) cutoff positions.

Figure 11. Power spectrum of the signal phase for out-of-focus (a) and in-focus (b) cutoff positions. 


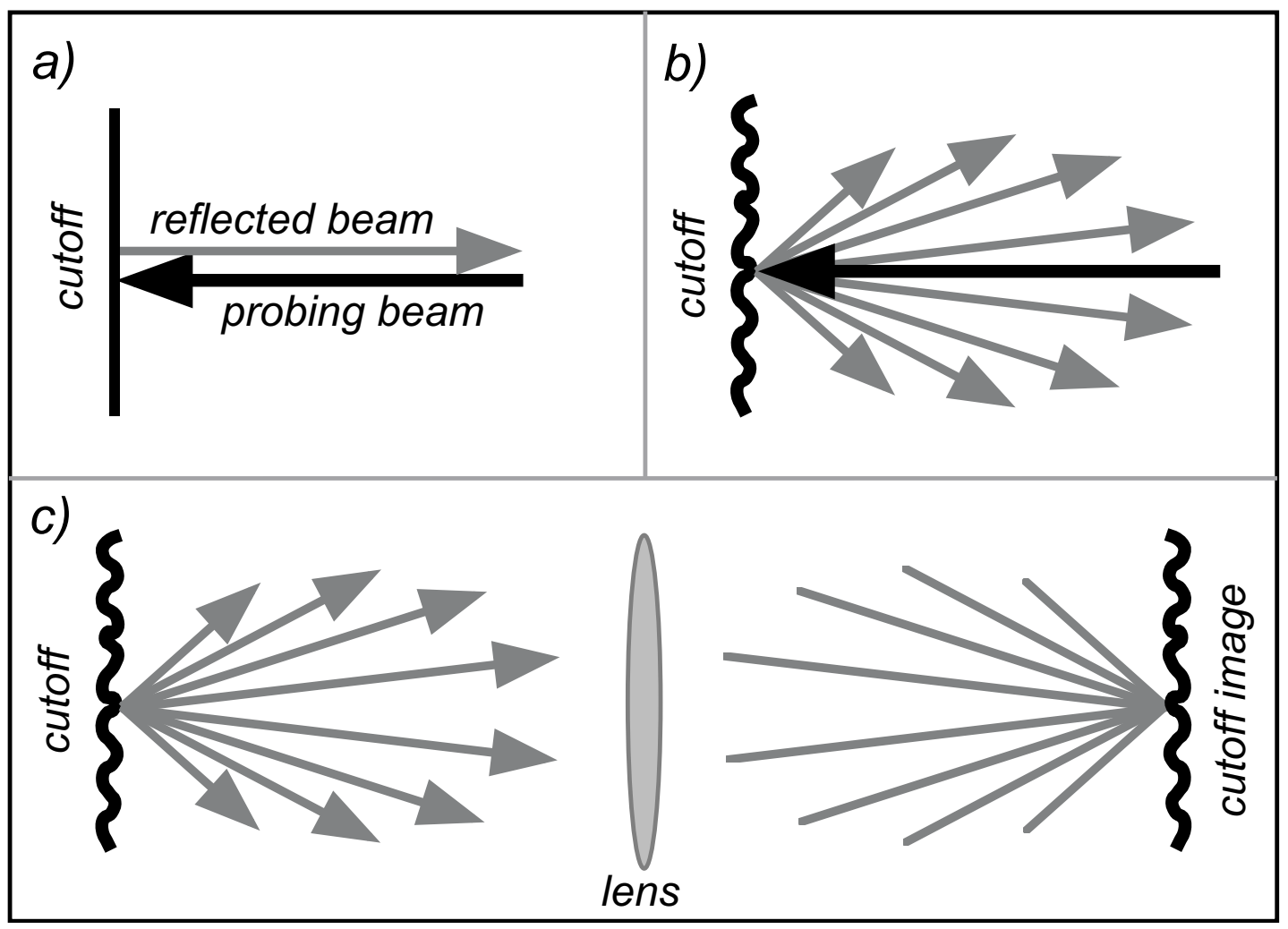

Figure 1. Schematic representation of standard reflectomery in 1D (a) and 2D (b) geometry, and that of imaging reflectometry $(c)$. 

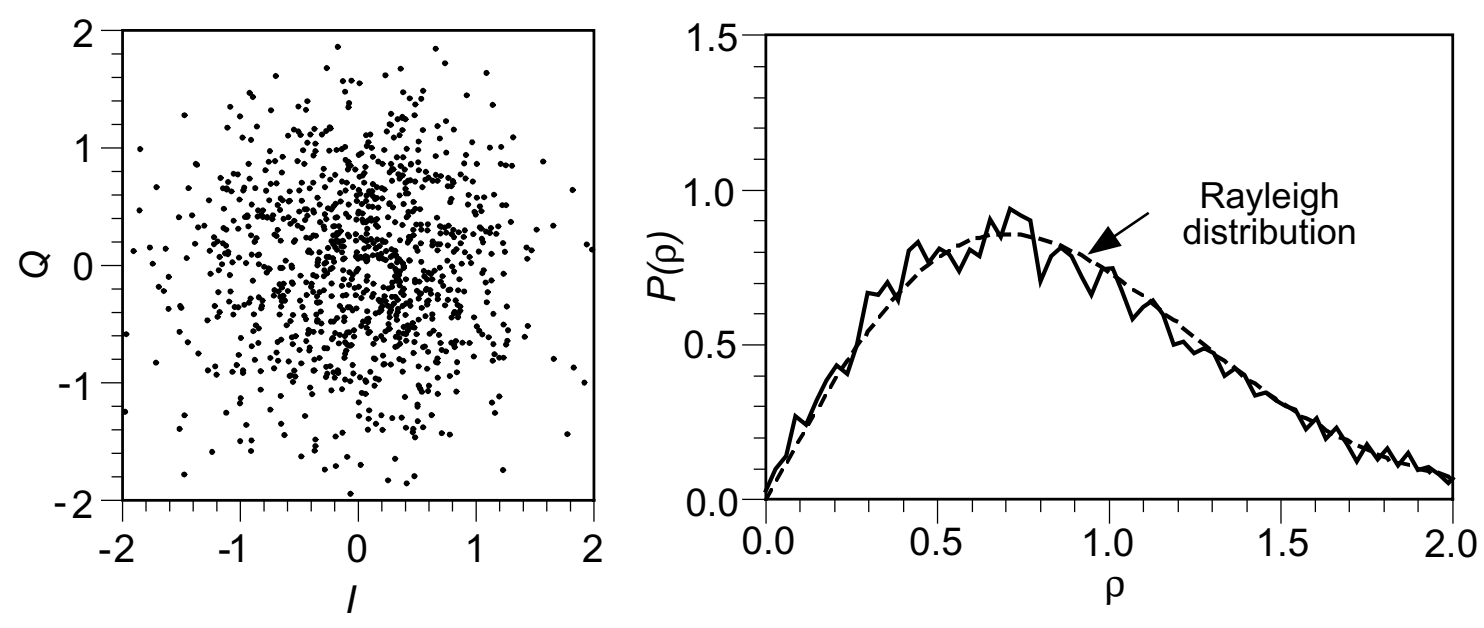

Figure 2. In-phase $(I)$ and quadrature $(Q)$ components of a TFTR reflectometer signal (left) and probability density distribution of the amplitude $\rho=\left(I^{2}+Q^{2}\right)^{1 / 2}$ (right). Dashed line is the Rayleigh distribution 

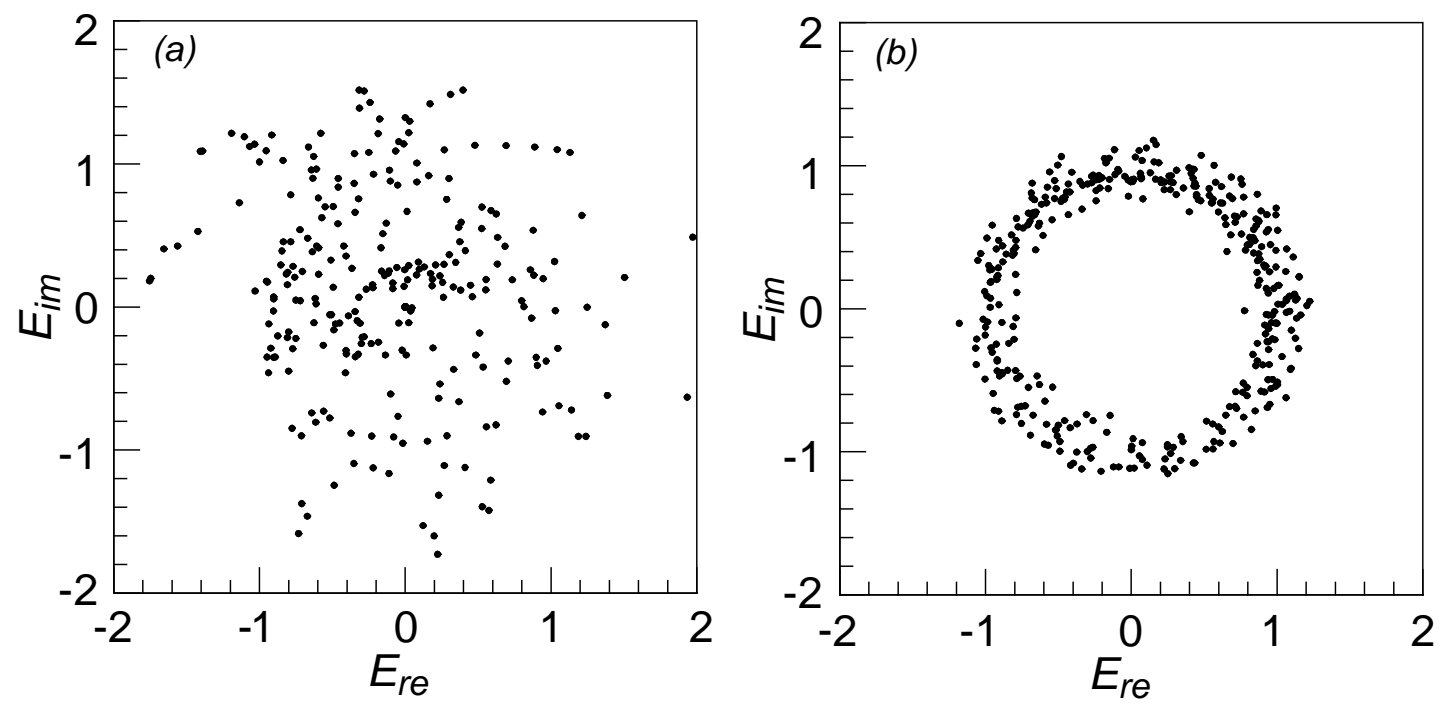

Figure 3. Plot of the complex amplitude of reflected waves at the plasma edge $(a)$ and at the virtual cutoff (b) for $\Delta k_{\theta}=0.5 \mathrm{~cm}^{-1}, \Delta k_{r}=1.0 \mathrm{~cm}^{-1}$, and $\tilde{n} / n=1.0 \%$ ). 

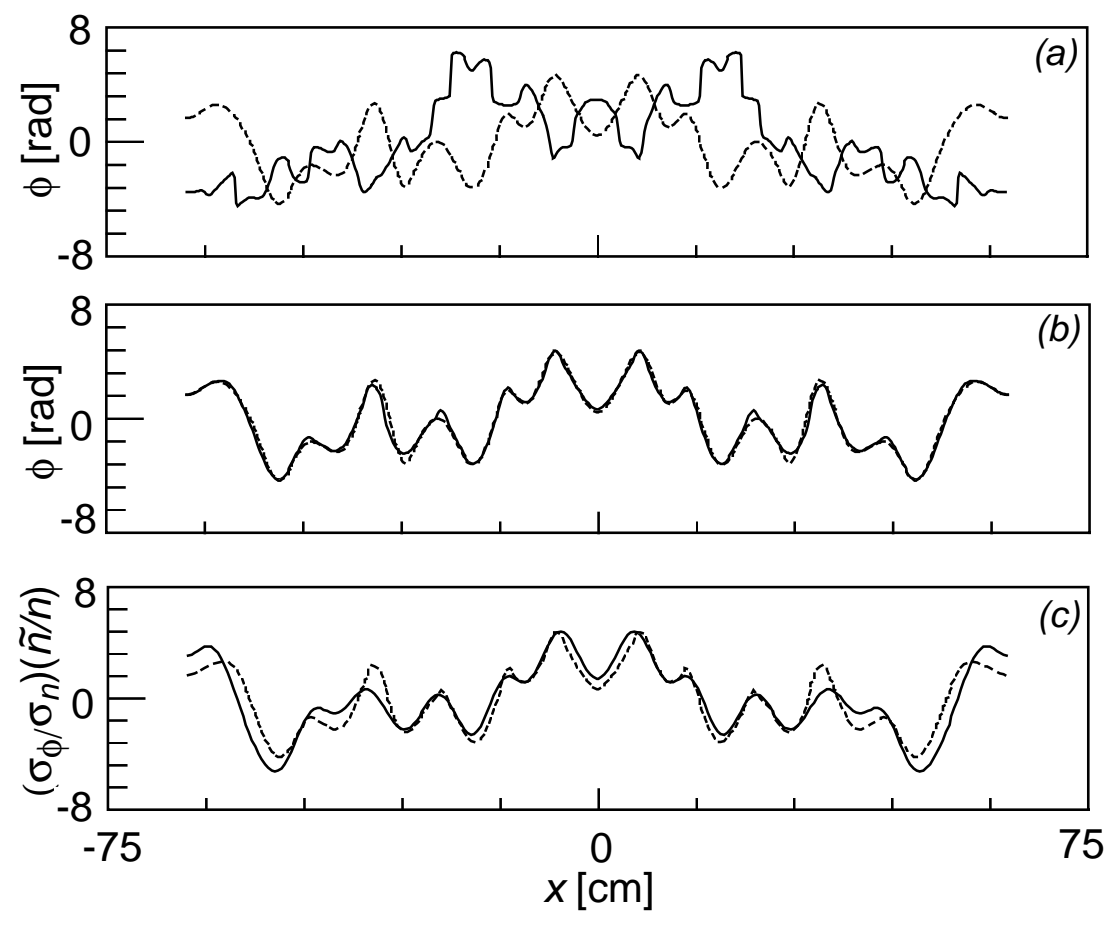

Figure 4. Phase fluctuation of reflected signals at the plasma boundary $(a)$ and the virtual cutoff $(b)$, and normalized density fluctuation near the cutoff $(c)$; dashed lines are the phase of 1D geometric optics $\left(\Delta k_{\theta}=0.5 \mathrm{~cm}^{-1}, \Delta k_{r}=1.0 \mathrm{~cm}^{-1}\right.$, and $\tilde{n} / n=1.0 \%$ ). 

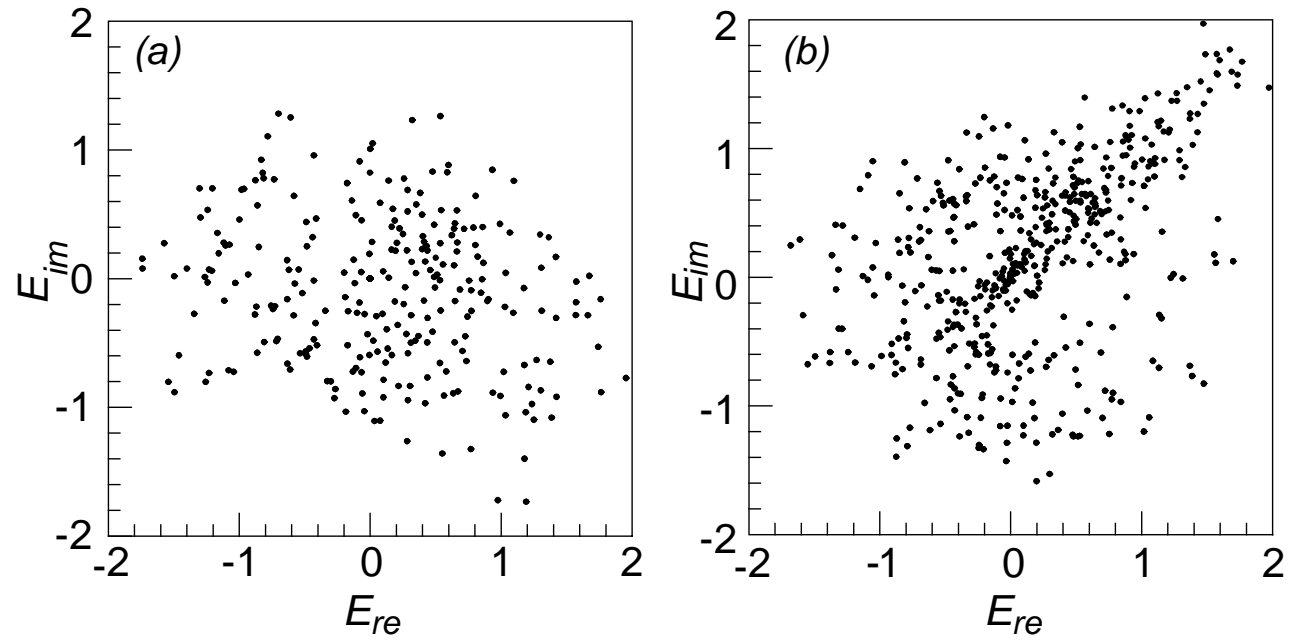

Figure 5. Plot of the complex amplitude of reflected waves at the plasma edge (a) and at the virtual cutoff $(b)$ for $L_{n}=50 \mathrm{~cm}, \Delta k_{\theta}=1.0 \mathrm{~cm}^{-1}, \Delta k_{r}=1.0 \mathrm{~cm}^{-1}$, and $\tilde{n} / n=1.5 \%$. 


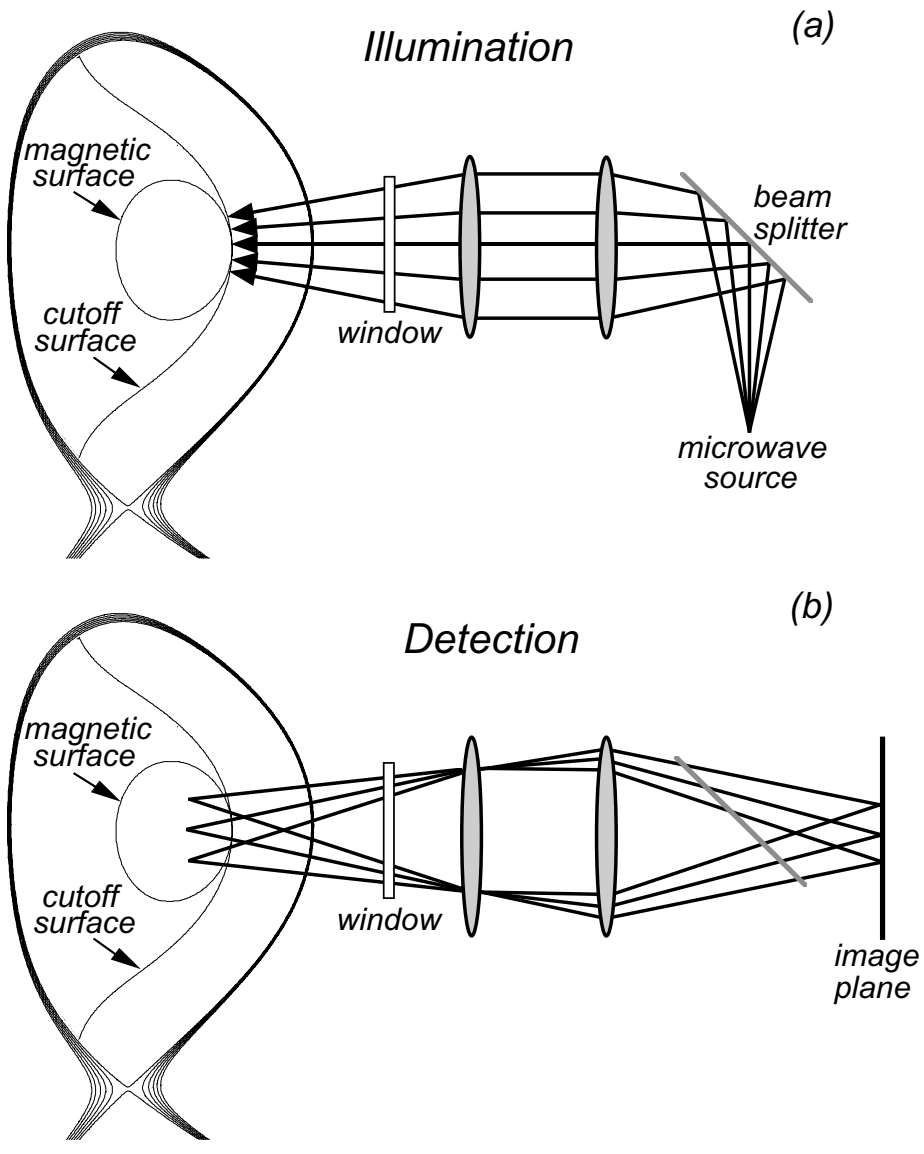

Figure 6. Schematic diagram of an imaging reflectometer showing probing (a) and reflected beams (b). 


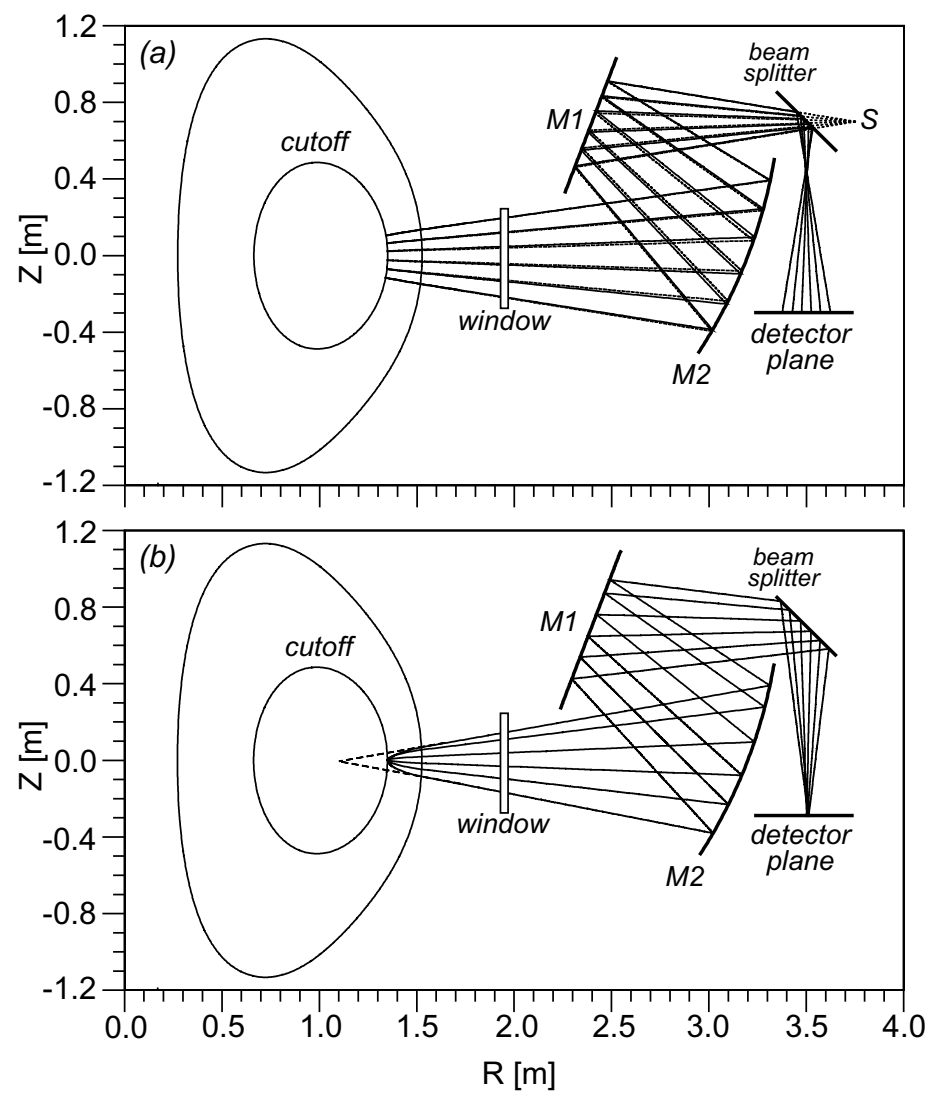

Figure 7. Conceptual design of an imaging reflectometer for NSTX; $\mathrm{S}$ is the microwave source, M1 and M2 are cylindrical mirrors. Ray trajectories are from a ray tracing code including plasma refraction. 

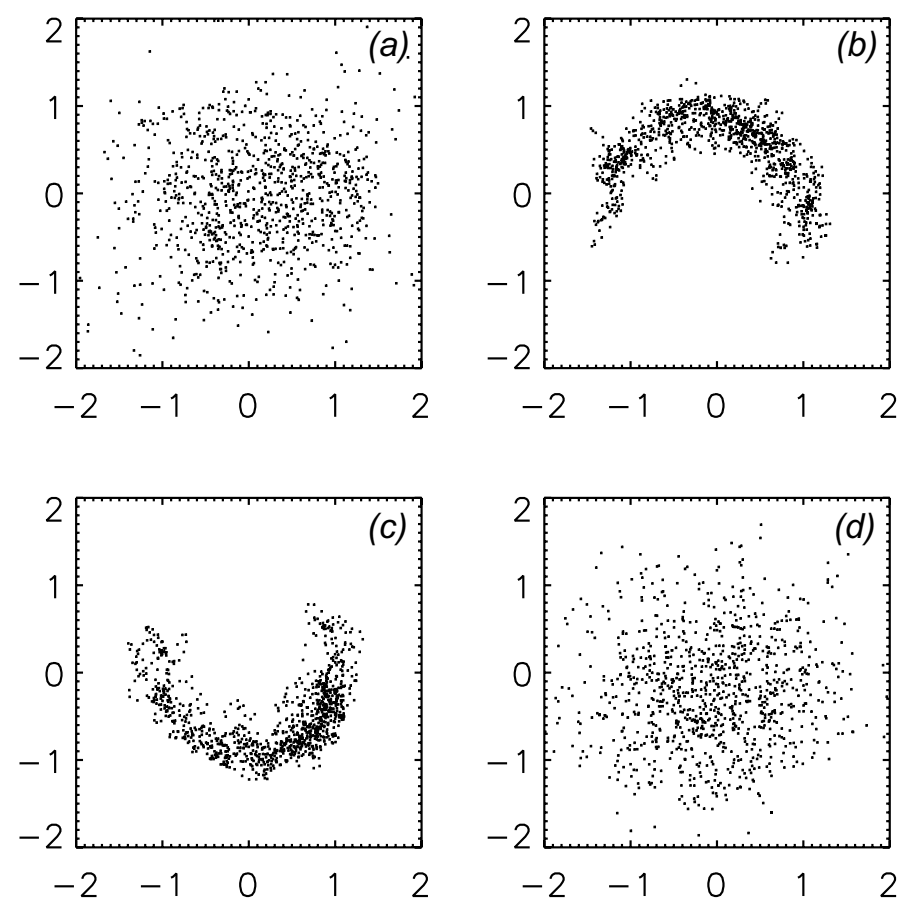

Figure 8. Quadrature signals plots (normalized to unit average power) over a $3 \mathrm{msec}$ time window as the cutoff moves through the optical focal plane because of a density rise. Cutoff positions are $1.93 \mathrm{~m}(a), 1.99 \mathrm{~m}(b, c)$, and $2.06 \mathrm{~m}(d)$. 

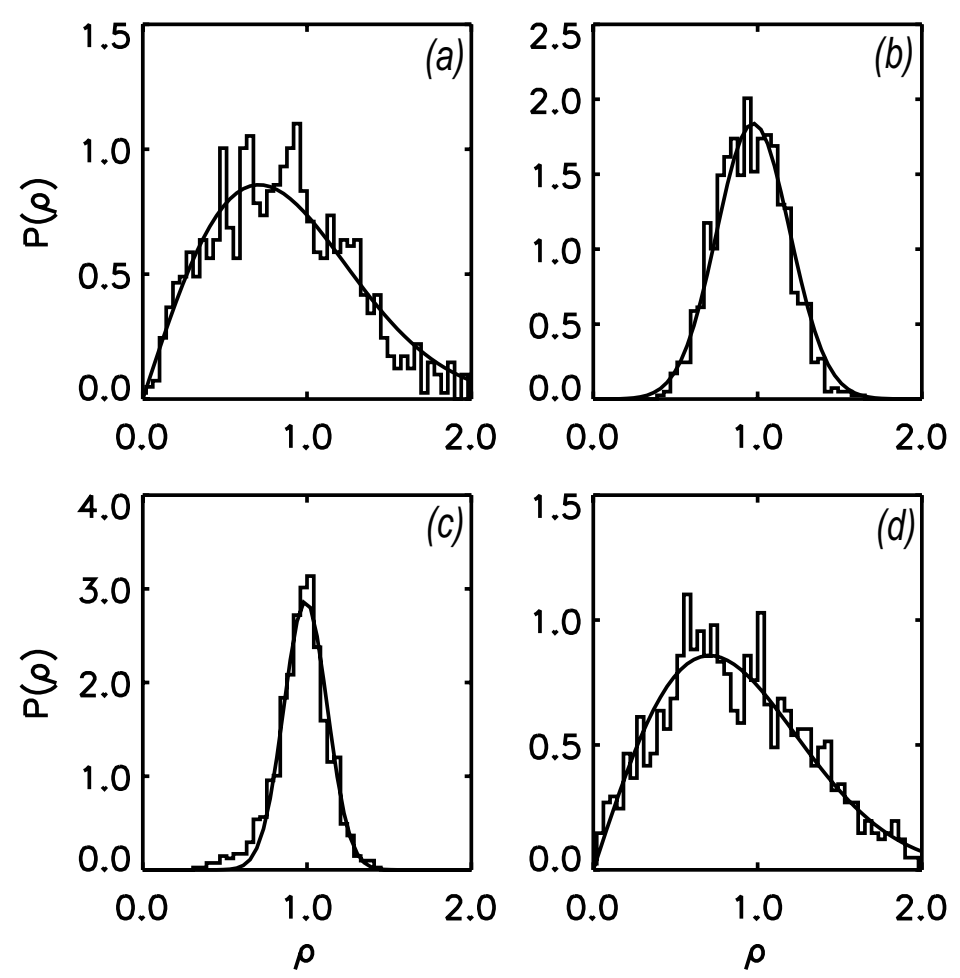

Figure 9. Amplitude histograms of the signals of Fig. 9 and best fit to a Rice distribution (smooth lines). The Rice parameters $\left[\rho_{0}, \sigma\right]$ are $[0.00,0.71](a),[0.95,0.22](b),[0.98$, $0.14](c),[0.00,0.71](d)$. 

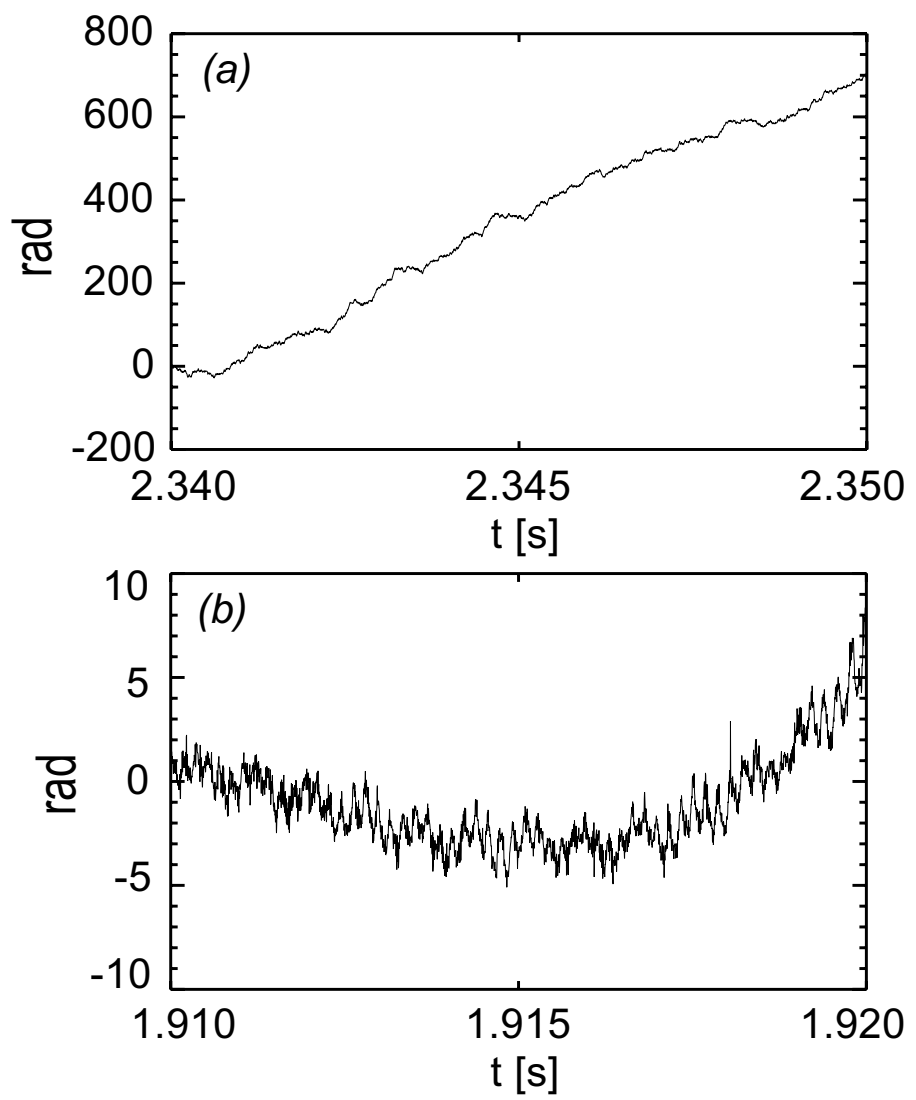

Figure 10. Time evolution of the signal phase during out-of-focus (a) and in-focus (b) cutoff positions 

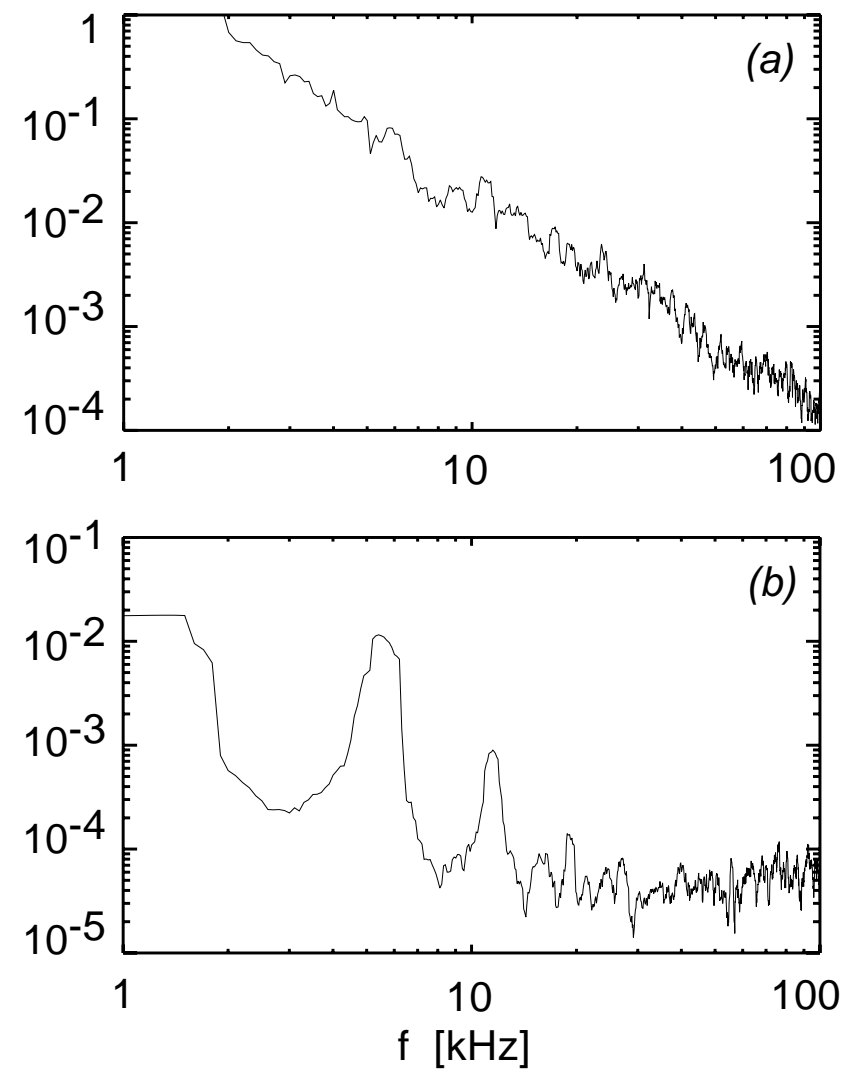

Figure 11. Power spectrum of the signal phase for out-of-focus (a) and in-focus (b) cutoff positions. 


\section{External Distribution}

Plasma Research Laboratory, Australian National University, Australia

Professor I.R. J ones, Flinders University, Australia

Professor J oão Canalle, Instituto de Fisica DEQ/IF - UERJ , Brazil

Mr. Gerson O. Ludwig, Instituto Nacional de Pesquisas, Brazil

Dr. P.H. Sakanaka, Instituto Fisica, Brazil

The Librarian, Culham Laboratory, England

Library, R61, Rutherford Appleton Laboratory, England

Mrs. S.A. Hutchinson, JET Library, England

Professor M.N. Bussac, Ecole Polytechnique, France

Librarian, Max-Planck-Institut für Plasmaphysik, Germany

J olan Moldvai, Reports Library, MTA KFKI-ATKI, Hungary

Dr. P. Kaw, Institute for Plasma Research, India

Ms. P.J . Pathak, Librarian, Insitute for Plasma Research, India

Ms. Clelia De Palo, Associazione EURATOM-ENEA, I taly

Dr. G. Grosso, Instituto di Fisica del Plasma, Italy

Librarian, Naka Fusion Research Establishment, J AERI, J apan

Library, Plasma Physics Laboratory, Kyoto University, J apan

Research Information Center, National Institute for Fusion Science, J apan

Dr. O. Mitarai, Kyushu Tokai University, J apan

Library, Academia Sinica, Institute of Plasma Physics, People's Republic of China

Shih-Tung Tsai, Institute of Physics, Chinese Academy of Sciences, People's Republic of China

Dr. S. Mirnov, TRINITI, Troitsk, Russian Federation, Russia

Dr. V.S. Strelkov, Kurchatov Institute, Russian Federation, Russia

Professor Peter Lukac, Katedra Fyziky Plazmy MFF UK, Mlynska dolina F-2, Komenskeho Univerzita, SK-842 15 Bratislava, Slovakia

Dr. G.S. Lee, Korea Basic Science Institute, South Korea

Mr. Dennis Bruggink, Fusion Library, University of Wisconsin, USA

Institute for Plasma Research, University of Maryland, USA

Librarian, Fusion Energy Division, Oak Ridge National Laboratory, USA

Librarian, Institute of Fusion Studies, University of Texas, USA

Librarian, Magnetic Fusion Program, Lawrence Livermore National Laboratory, USA

Library, General Atomics, USA

Plasma Physics Group, Fusion Energy Research Program, University of California at San Diego, USA

Plasma Physics Library, Columbia University, USA

Alkesh Punjabi, Center for Fusion Research and Training, Hampton University, USA

Dr. W.M. Stacey, Fusion Research Center, Georgia Institute of Technology, USA

Dr. J ohn Willis, U.S. Department of Energy, Office of Fusion Energy Sciences, USA

Mr. Paul H. Wright, Indianapolis, Indiana, USA 
The Princeton Plasma Physics Laboratory is operated by Princeton University under contract with the U.S. Department of Energy.

\author{
Information Services \\ Princeton Plasma Physics Laboratory \\ P.O. Box 451 \\ Princeton, NJ 08543
}

Phone: 609-243-2750

Fax: 609-243-2751

e-mail: pppl_info@pppl.gov

Internet Address: http://www.pppl.gov 